Supporting Information

\title{
Phenotype Profiling for Forensic Purposes: Determining Donor Sex based on Fourier Transform Infrared Spectroscopy of Urine Traces
}

\author{
Ayari Takamura $^{1,2}$, Lenka Halamkova $^{3}$, Takeaki Ozawa ${ }^{1 *}$, Igor K. Lednev ${ }^{3 * *}$ \\ ${ }^{1}$ Department of Chemistry, Graduate School of Science, The University of Tokyo \\ 7-3-1, Hongo, Bunkyo, Tokyo 113-0033, Japan \\ ${ }^{2}$ First Department of Forensic Science, National Research Institute of Police Science \\ 6-3-1, Kashiwanoha, Kashiwa, Chiba 277-0882, Japan \\ ${ }^{3}$ Department of Chemistry, University at Albany, SUNY \\ 1400 Washington Avenue, Albany, New York 12222, United States
}

Correspondence should be addressed to:

Takeaki Ozawa (*)

E-mail: ozawa@chem.s.u-tokyo.ac.jp

Tel.: +81-3-5841-4351, Fax: +81-3-5802-2989

Igor K Lednev $(* *)$

E-mail: ilednev@albany.edu

Tel.: +1-518-591-8863, Fax: +1-518-442-3462 
(A)

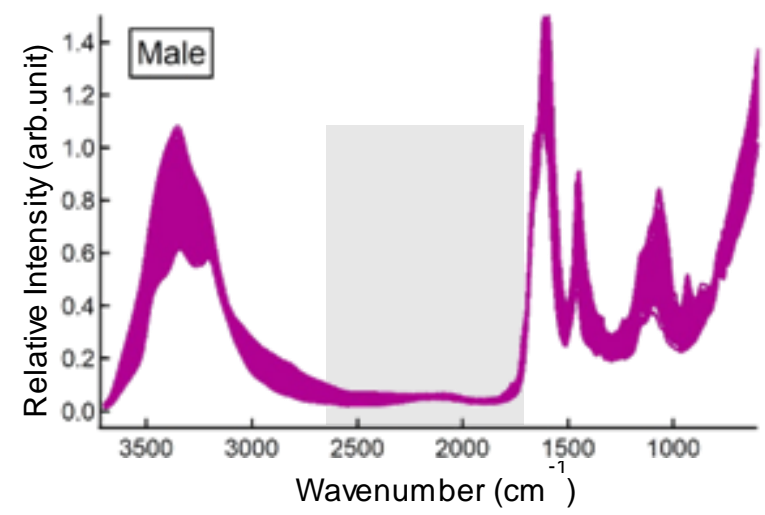

(C)

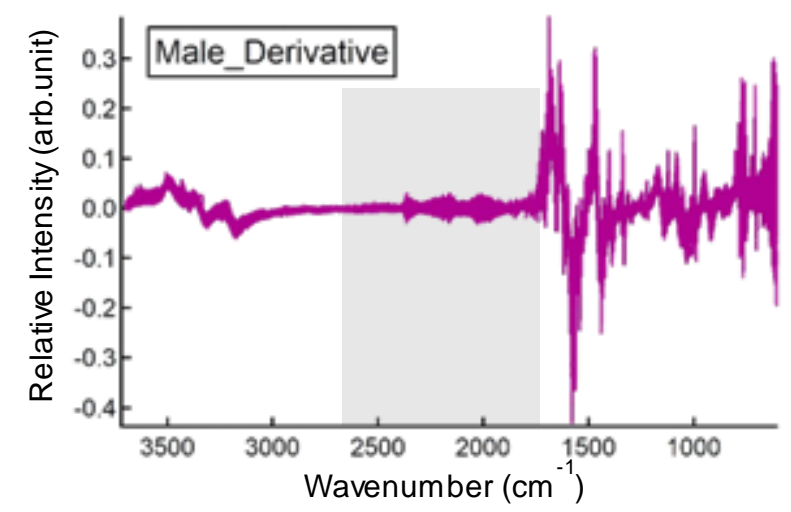

(B)

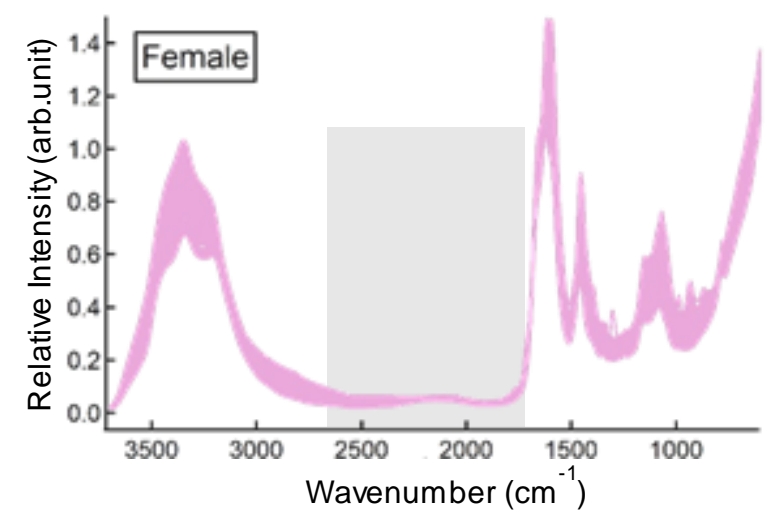

(D)

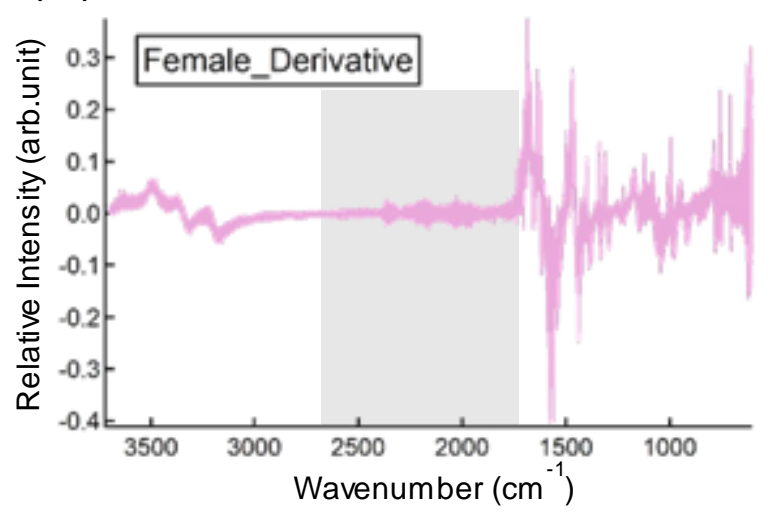

Figure S-1. Spectral variations among the ATR FT-IR spectra of urine. Collected individual ATR FT-IR spectra of urine (A, B) and the individual derivative spectra (C, D) are overlaid for the male (A, C) and female (B, D) samples, respectively. Each spectrum was normalized by the total area. Gray regions in the graphs indicate the spectral ranges excluded prior to the multivariate analysis, due to the crystal interference of the ATR FT-IR instrument. 

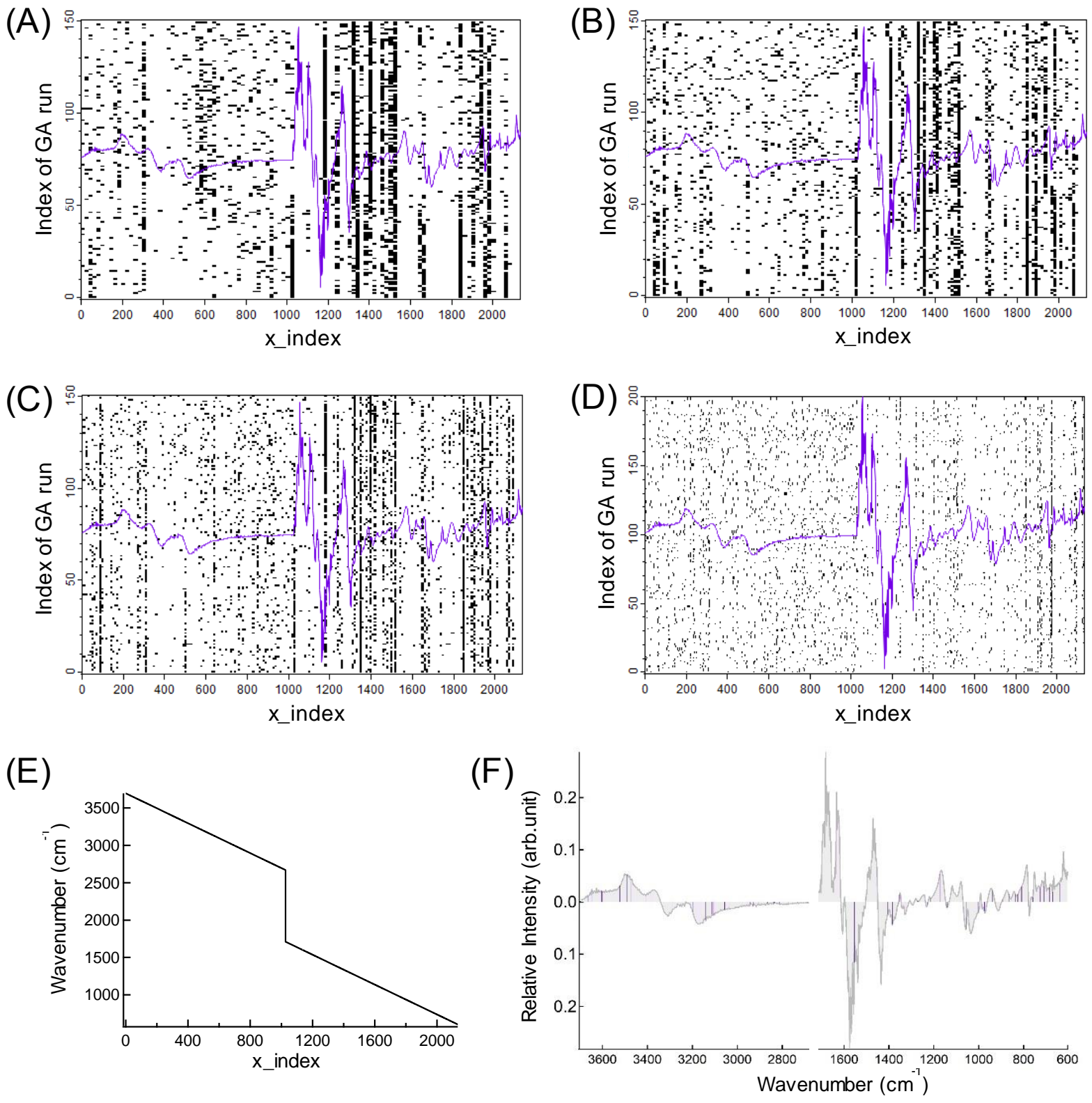

Figure S-2. Genetic Algorithm (GA) for differentiating the donor sex of urine spectra. The GA was implemented with wavenumber block sizes of $20 \mathrm{~cm}^{-1}$ (A), $15 \mathrm{~cm}^{-1}$ (B), $10 \mathrm{~cm}^{-1}$ (C) and $1 \mathrm{~cm}^{-1}$ (D). The number of runs implemented in each GA condition was 150 or 200. The selected significant spectral regions in each run were represented with black, in order of PRESS at stop of each run from minimum (the bottom) to max (the top) (Index of GA run). The averaged derivative spectra of urine, except the region of 1711-2669 $\mathrm{cm}^{-}$ ${ }^{1}$, were shown with purple lines. (E) Alternative variables, “x_index", used to represent corresponding wavenumbers in Figure S-2(A)-(D). (F) The best solution identified by the GA (purple regions), which was obtained with the wavenumber block size of $1 \mathrm{~cm}^{-1}$. 
(A)
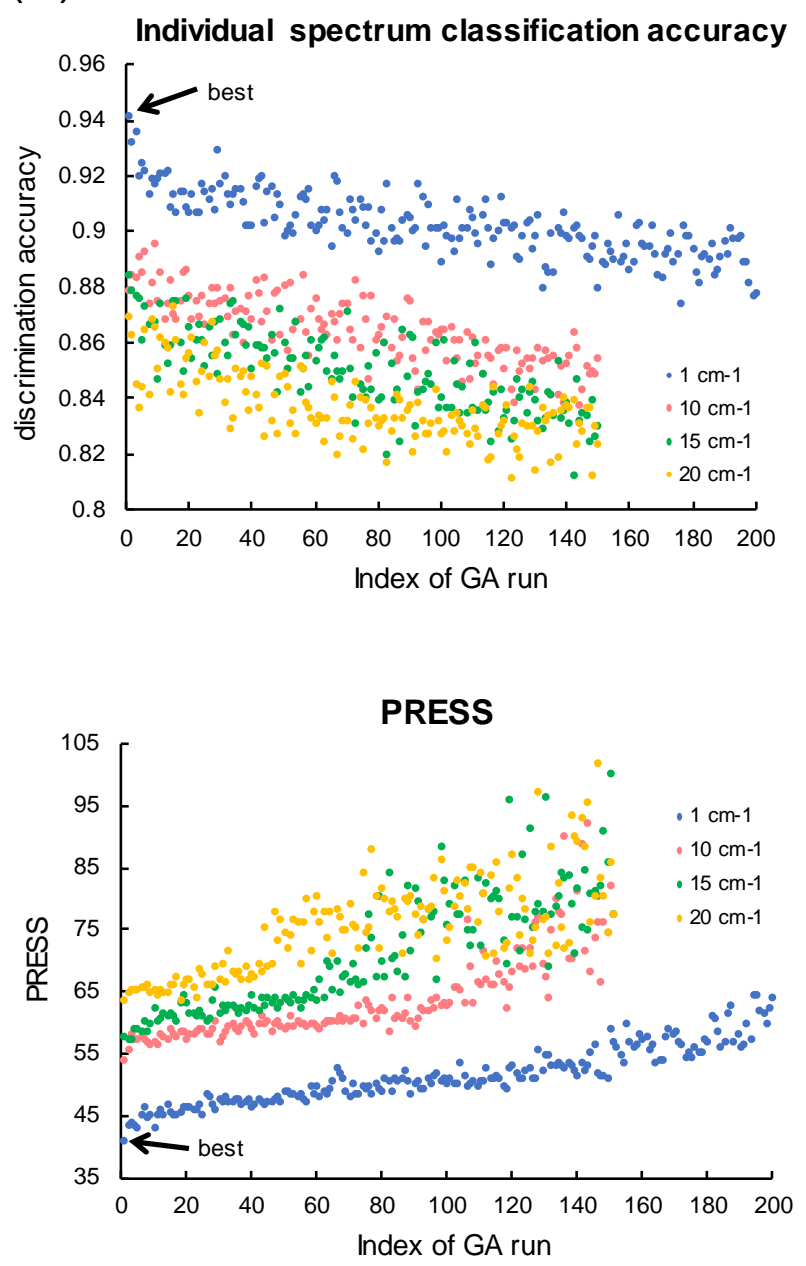

(B)

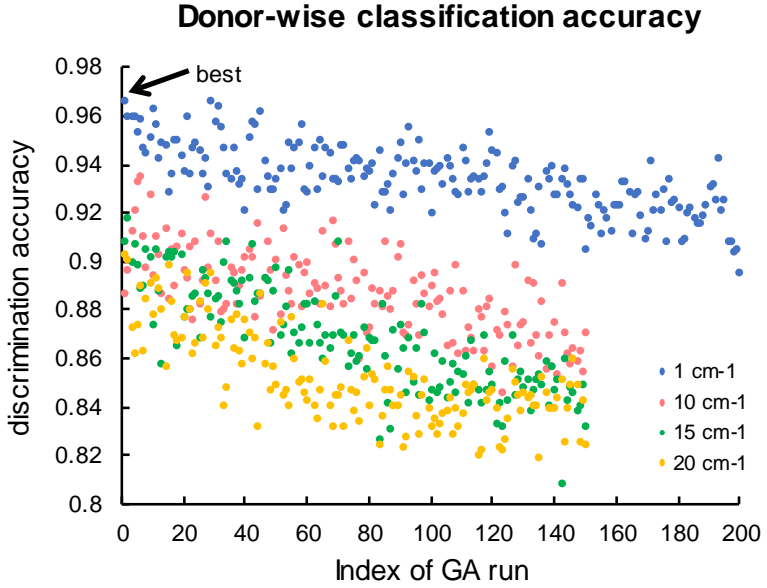

(C)

Figures S-3. Evaluation of PLS-DA models built with variables selected by GA. The spectral regions selected by each GA run with the wavenumber block size of 20,15, 10 or $1 \mathrm{~cm}^{-1}$ were applied to the PLS-DA modeling for donor sex differentiation of urine spectra. The performance of the developed model was evaluated by ten times repeats of 10 -fold CV. Then, averaged discrimination accuracies for individual spectrum classification (A) and donor-wise classification (B) and PRESS values (C) were obtained. Each of the calculated values was plotted in order of PRESS at stop of each GA run, from minimum to max (Index of GA run, corresponding to Figure S-2 (A)-(D)). Dots with yellow, green red and blue indicate the results of the GA runs with the wavenumber block sizes of $20,15,10$ and $1 \mathrm{~cm}^{-1}$, respectively. The identified best solution was represented with an arrow in each graph. 
(A)

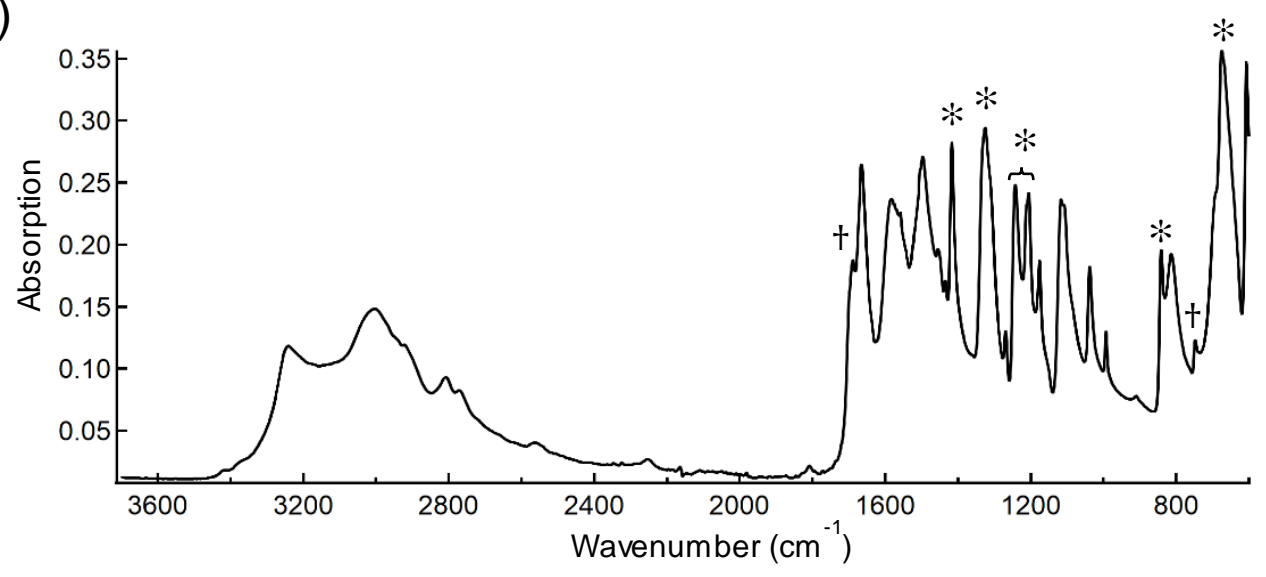

(B)

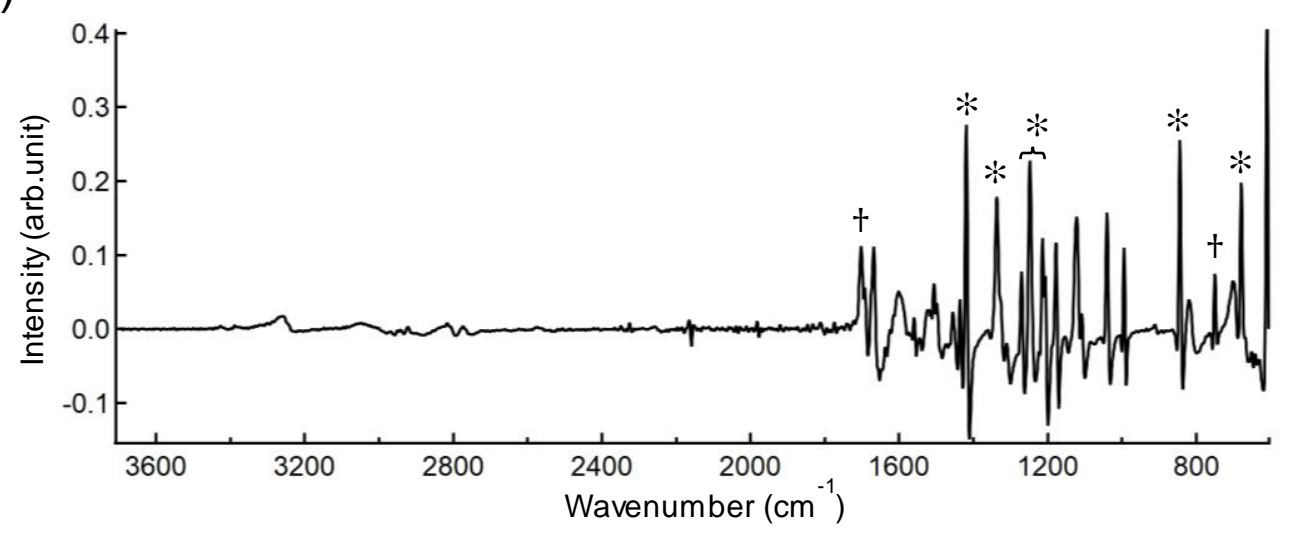

Figure S-4. Reference ATR FT-IR spectrum of creatinine. (A) Raw ATR FT-IR absorption spectrum of creatinine crystals. (B) First-order derivative spectrum of creatinine, calculated by the second polynomial Savitzky-Golay method. "* GA for the urine spectra. " $\uparrow$ " $s$ indicate the relatively minor peaks which possibly correspond to the selected regions by the GA. 


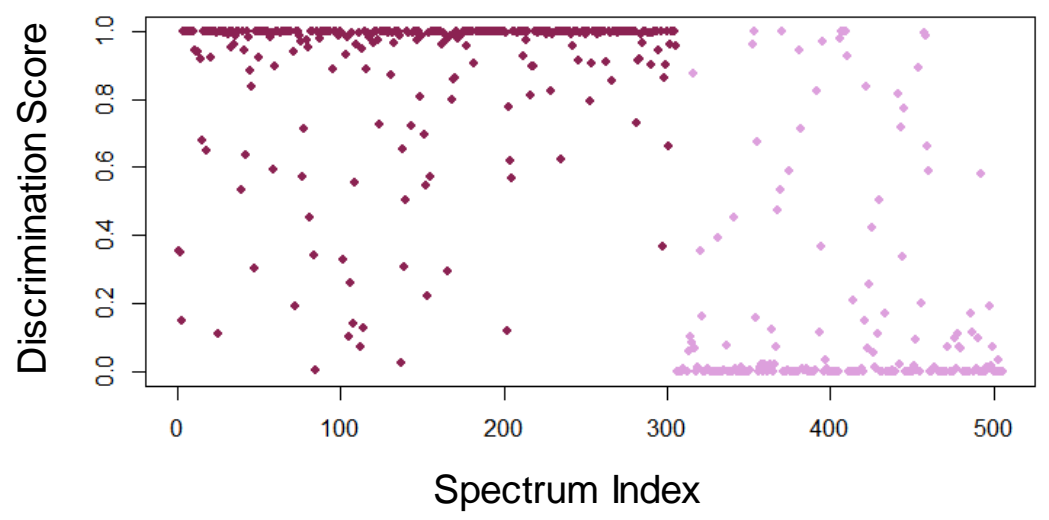

Figure S-5. Discrimination scores for donor sex determination of the urine spectra, predicted by an artificial neural network (ANN) method coupled with the GA-selected wavenumbers. The ANN model was built and validated by donor-independent 10 -fold $\mathrm{CV}$. Each dot indicates the individual spectral data of male (magenta) and female (pink) urine. 
Table S-1. Discrimination performance of the ANN model for discriminating the donor sex of urine spectra. $^{a}$

\begin{tabular}{ccccc}
\hline \multirow{2}{*}{ Model } & Spectrum & $\begin{array}{c}\text { True Male rate } \\
\text { (specificity) }\end{array}$ & $\begin{array}{c}\text { True Female rate } \\
\text { (sensitivity) }\end{array}$ & Total Accuracy \\
\hline \multirow{2}{*}{ ANN-GA } & Single & $0.947 \pm 0.010$ & $0.898 \pm 0.022$ & $0.928 \pm 0.009$ \\
\cline { 2 - 5 } & Donor & $0.99 \pm 0.01$ & $0.93 \pm 0.03$ & $0.96 \pm 0.01$ \\
\hline
\end{tabular}

${ }^{a} \overline{\text { Each value was evaluated by } 100 \text { repeats of donor-independent } 10 \text {-fold CV (average } \pm \text { standard deviation). }}$ 